\title{
Attention Drawing of Movie Trailers Revealed by Electroencephography Using Sample Entropy
}

\author{
Po-Shan Wang1, Shang-Ran Huang'2, Chao-Wen Tsai ${ }^{2}$, Chia-Feng Lu'², Shin Teng2, \\ C.-I. Hung' ${ }^{2}$, Yu-Te $\mathrm{Wu}^{2,3}$ \\ ${ }^{1}$ The Neurological Institute, Taipei Municipal Gan-Dau Hospital, New Taipei, Chinese Taipei \\ ${ }^{2}$ Department of Biomedical Imaging and Radiological Sciences, National Yang-Ming University, Taipei, \\ Chinese Taipei \\ ${ }^{3}$ Institute of Biophotonics, National Yang-Ming University, Taipei, Chinese Taipei \\ Email: ytwu@ym.edu.tw
}

Received February 2014

\begin{abstract}
A movie trailer is a common advertising tool in the entertainment industry. Detection of a viewer's brain responses to a movie trailer can help film producers to tailor a more appealing trailer of a movie. In this study, we acquired electroencephalographic (EEG) signals from subjects when they watched movie trailers (labeled as Movie session), and compared with their resting state session (labeled as Resting session) or when they watch nature scenes (labeled as Nature session). We used Sample Entropy (SampEn) to analyze the EEG signals between different sessions. Results showed that the complexity ratios at Fp1, Fp2 and Fz channels derived from Movie session were significantly lower than that in Resting state or when subjects watched Nature session $(p<0.001)$. Our results suggest that the brain status can affect the complexity of their EEG. Further, the attraction of attention of a movie trailer can be observed from the change of EEG.
\end{abstract}

\section{Keywords}

Neuromarketing, Electroencephalography, Sample Entropy, Movie Trailer

\section{Introduction}

Watching movies is a part of life in modern society. The scale of this entertainment industry has been over 10 billion US dollars since 2009 in North American market alone [1]. However, not every movie can generate positive income for the film producers and publishers. A successful promotional campaign plays a key role in boosting the box office and resulting in positive net gross of a movie. With easy accessibility to movie database through internet, such as YouTube, Rotten Tomatoes and Internet Movie Database (IMDb), movie trailers have become a powerful advertising tool to promote a movie. In 2008, movie trailers clinched the third place on the list of the most watched online videos [2]. Therefore, how to produce a good trailer for each movie become 
more and more important for each movie producers.

In neuromarketing [3], modern techniques, such as electroencephalography (EEG), magnetic resonance imaging (MRI), near infrared spectroscopy (NIRS) and so on, are used to investigate a customer's cerebral feedback to an advertisement. An American multinational neuromarketing company, NeuroFocus, stresses on using three primary neurometrics (attention, memory and emotion) and three secondly derived neurometrics (purchase intent, novelty and awareness) to assess the effectiveness of the advertisement [4]. Several reports have been proposed to investigate a customer's cerebral response to different advertising static pictures or television commercials [5]-[7].

In contrast to the static pictures and television commercials films, few studies had focused on movie trailers before. It could be because movie trailers are more complicated to study when compared to the television commercial films. A movie trailer tends to have longer duration, and its advertising effectiveness is influenced by variety of factors like movie genres, cast, director, etc. In this study, we focused on whether a movie trailer can draw viewers' attention so that the brain status change can be detected by using modern EEG techniques.

\section{Materials and Methods}

\subsection{Data Acquisition}

Twenty-seven right-handed health volunteers were recruited in this study, including 14 males and 13 females. Their average age was $24.9 \pm 3.2$ (range 21 - 31). All of them were Mandarin Chinese native speakers. All the subjects had normal or corrected-to-normal vision. None of them had neurological or psychiatric illness or alcohol abuse, and all of them were medication-free. Before the experiment, the purpose of this study was explained to the subjects. Informed consent was obtained from each subject. This study was performed based on the declaration of Helsinki and approved by Institutional Review Board of National Yang-Ming University, Chinese Taipei.

A 40 channel QuickAmp system (Brain Products GmbH, Gilching, Germany) was used to record the EEG signals, and the sampling rate was $500 \mathrm{~Hz}$. Based on the International 10 - 20 system, six EEG electrodes were used at the following recording sites, namely, Fp1, Fp2, Fz, O1, O2 and Oz, with the ground at AFz referred to common reference in this study (Figure 1). In addition, two additional bipolar electrooculogram (EOG) was used to detect eye movements; and the other two EEG electrodes were attached to the left and right ear lobe separately from which average signals of both ear lobes were used as reference. The EEG electrode impedances were kept below $5 \mathrm{k} \Omega$ in most cases. Experimental stimuli were displayed on the screen of a personal computer (PC) with synchronization markers sent to the recording laptop. We used the software "Presentation" by Neurobehavioral Systems for displaying the stimuli. Subjects underwent the experiment in a light-controlled room and sat in a comfortable office chair. Also, subjects were instructed to keep awake and relaxed for entire duration in the experiment. The subjects sat about $100 \mathrm{~cm}$ away from the stimuli displaying PC. They were asked to sit quietly and to look at the screen to minimize the muscle and eye movement artifacts.

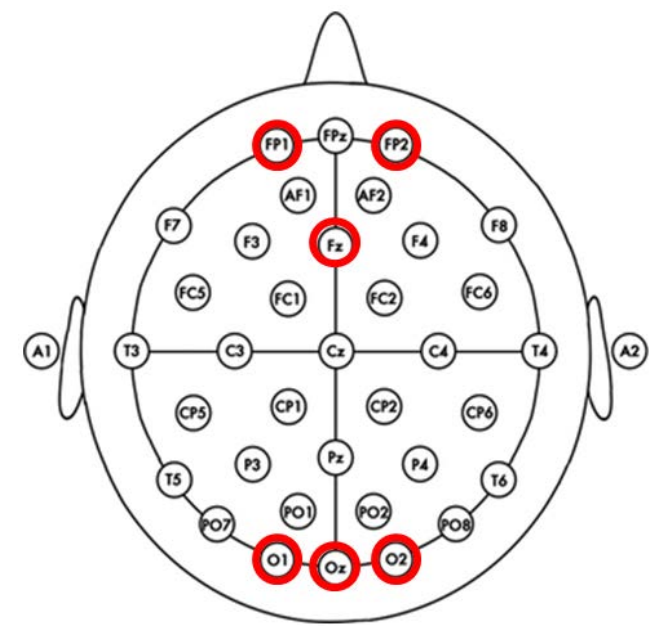

Figure 1. EEG electrodes montage. Red circles indicate the 6 electrodes, Fp1, Fp2, Fz, O1, O2, and Oz, used in the experiment. 


\subsection{Paradigm}

In the experiment, each subject needed to watch 5 video clips, including 1 natural scenery video and 4 movie trailers which were coming soon to theaters. The study totally consisted of 7 sessions. The procedure is illustrated in Figure 2. At the beginning, the subjects were asked to focus on watching a fixation white cross on a black background without thinking anything; and the cross was presented in the center of the computer screen. The session was called "Resting 1". After the Resting 1 session, the natural scenery video was played accompanied by soft piano music, and we called this session as "Nature". Following the natural scenery video clip, four different movie trailers were presented and the time duration of trailers varied for each one. We named those sessions of movie trailers as "Movie 1", "Movie 2", "Movie 3" and "Movie 4" in order. The subjects might see different movie trailers, but all the four trailers were not on theater before the date of the experiment. The release dates for these trailers selected in this study were between Sept. 2011 and Aug. 2012. The collection of selected trailers included different movie genres such as comedy, romance, animation, action, etc. Moreover, the movies were from several different countries, but all the movie trailers had Traditional Chinese subtitles. Finally, a session analogous to Resting 1 was added to the end of the experiment. The subjects had to look at a fixation white cross again and not to think about anything as what they did in Resting 1 . The second resting session was called "Resting 2" and the duration was 3 minutes, too. There were 10-sec rests between sessions and a cue was displayed on the screen before the video clips played.

\subsection{Data Analysis}

We used sample entropy (SampEn) [8] to analyze the EEG signal. SampEn is based on the concept to measure the randomness of a time series. We denote the SampEn of an $N$-length time series as SampEn $(m, r, N)$, and it depends on three parameters: $\mathrm{m}$ is embedded dimension; $\mathrm{r}$ is tolerance; and $N$ is estimated data length. Given a time series $X$ of length $n, X_{i}=\left\{x_{1}, x_{2}, \ldots, x_{n}\right\}$. The value of SampEn is computed by the following process:

1. Let $l=m$.

2. Form two set of vectors:

$$
\begin{gathered}
u_{m}(i)=\left\{x_{i}, x_{i+1}, \ldots, x_{i+m-1}\right\}, 1 \leq i \leq N-l \\
u_{m}(j)=\left\{x_{j}, x_{j+1}, \ldots, x_{j+m-1}\right\}, 1 \leq j \leq N-l
\end{gathered}
$$

3. Define distance between two vectors from sets (1) and (2) as:

$$
d\left[u_{m}(i), u_{m}(j)\right]=\max _{k=1, \ldots, m}(\operatorname{absolute}(u(i+k-1)-u(j+k-1)))
$$

4. Define $n_{i}^{m}(r)$ as the number of vectors $u_{m}(j)$ which distance was within $r$ of $u_{m}(i)$, where $j \neq i$.

5. Define $B_{i}^{m}(r)=n_{i}^{m}(r) /(N-m-1)$ and calculate the average of $B_{i}^{m}(r)$ for all $i$ as:

$$
B^{m}(r)=\frac{1}{N-m} \sum_{i=1}^{N-m} B_{i}^{m}(r)
$$

6. Change the dimension $m$ to $m+1$, repeat step $2-5$ and similarly calculate

$$
A^{m}(r)=\frac{1}{N-m} \sum_{i=1}^{N-m} A_{i}^{m}(r)
$$

7. $\operatorname{SampEn}(m, r, N)=-\ln \left[A^{m}(r) / B^{m}(r)\right]$

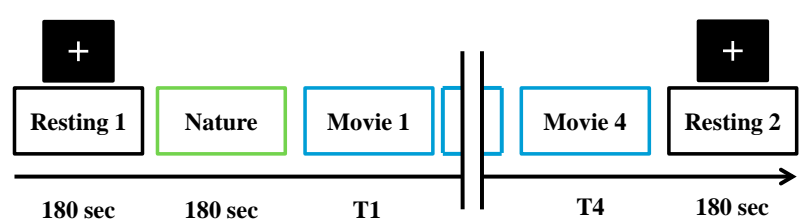

Figure 2. Flowchart of experiment. 
Assume the data series $X=\left\{x_{1}, x_{2}, \ldots, x_{n}\right\}$ was the EEG recoding of one channel in one of the experimental session, and $X$ was filtered out the $60 \mathrm{~Hz}$ component. We calculated the SampFn for every second, that is, 500 data points, because of the sampling frequency of the EEG system was $500 \mathrm{~Hz}$. We set $m=2, r=0.15$ and $N=$ 500 and calculated the value of SampEn for each second and obtain a set $Y=\left\{\operatorname{SampEn}\left(x_{1} \ldots x_{500}\right), \ldots\right.$, Sam$\left.\operatorname{pEn}\left(X_{(n-500)+1} \ldots x_{n}\right)\right\}$, in which the number of data was 1/500 times of the data length of each EEG recording $X$. For example, if the data $X$ in the resting state was 180 seconds, i.e., $n=500 \times 180$, the number of resultant SampEn values was 180. We computed the distribution of the SampEn values in different 7 sessions of each channel. There was a peak for the SampEn value between 2 and 3, especially in Fp1, Fp2 and Fz; and this pattern was not observed in occipital channels. We computed the ratio of the number of the SampEn values in $Y$ which were between 2 and 3 to the total data number of $Y$, with only Fp1, Fp2 and Fz considered.

$$
\text { Complexity ratio }=\frac{\text { the number of the SampEn in } Y \text { which were between } 2 \text { to } 3}{\text { total data number of } Y}
$$

The complexity ratios in whole 7 sessions of all subjects in Fp1, Fp2 and Fz were calculated and analyzed by the Statistical Package for the Social Science (SPSS) 18.0.

\section{Results}

Figure 3 showed that the mean values of EEG complexity ratios in the 2 Resting sessions, the Nature session and the 4 Movie sessions were apparently different. Different to the Resting and Nature sessions, the mean complexity ratio declined significantly when subjects watched the 4 Movie sessions. The mean complexity ratios of the Nature session were higher than Movie sessions but lower than the Resting 1 and Resting 2 sessions. The mean complexity ratio at both of the Resting 1 and Resting 2 sessions were highest in all sessions.

In order to examine whether the complexity ratios among different sessions were significantly different, we compared the Resting 1 sessions with other sessions, Nature session with other sessions, and any session with its adjacent session using the paired t-test. Results were presented in Table 1. Results demonstrated that the com-
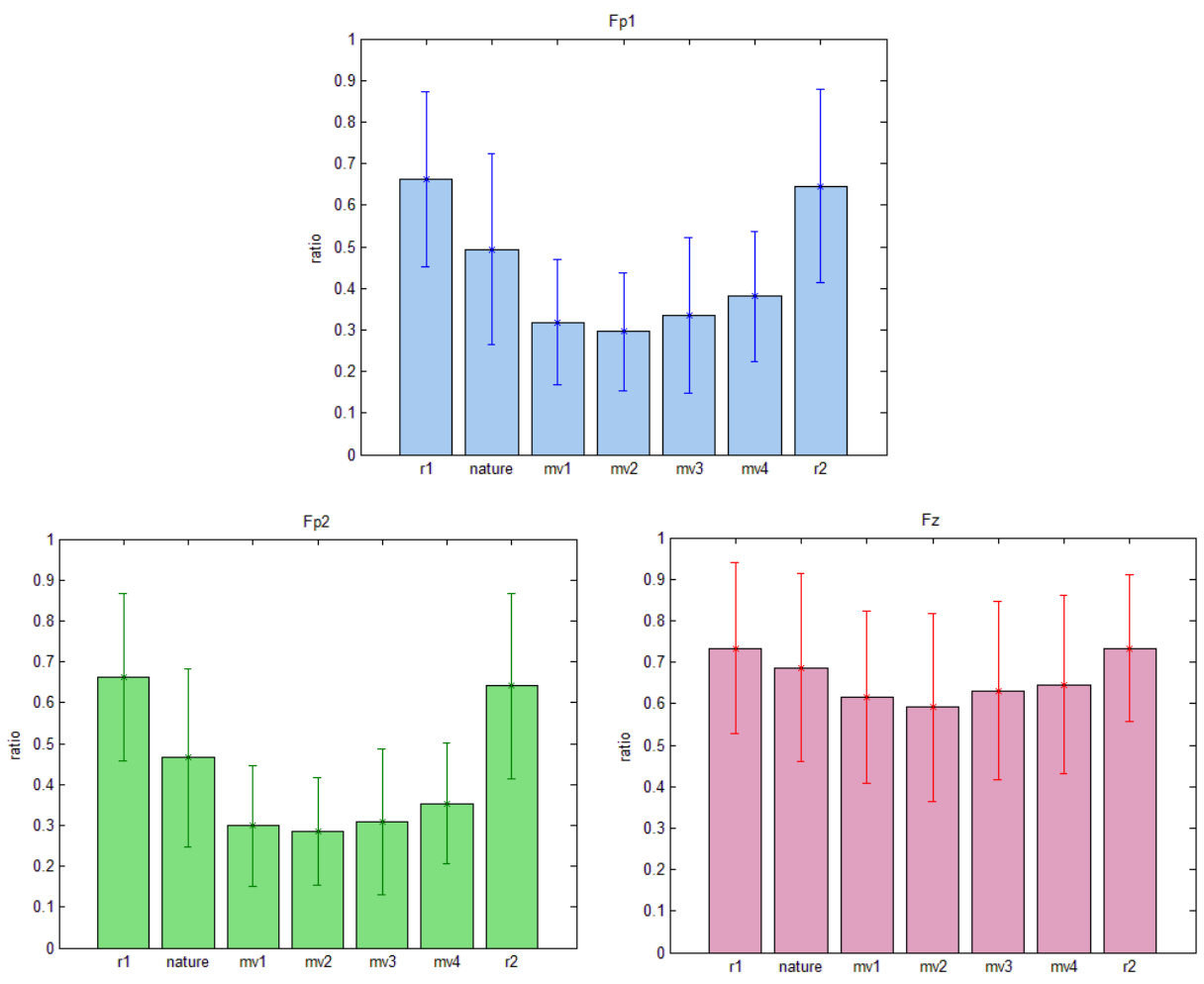

Figure 3. The mean and standard deviation of complexity ratio in the 7 sessions. Horizontal axis: 7 sessions. Left to right: r1, nature, mv1, mv2, mv3, mv4, r2 (r1 and r2 stand for resting 1 and resting 2; mv 1 - 4 stands for movie 1 - 4). Vertical axis: the value of complexity. Top to bottom: Fp1 (blue), Fp2 (green) and Fz (pink). 
Table 1. Statistical comparison of complexity ratios between different sessions. r1 and r2 stand for resting 1 and resting 2; mv 1 - 4 stands for movie 1 - 4. Top: compared with Resting 1. Middle: compared with Nature. Bottom: compared with previous session. ${ }^{*}: \mathrm{p}<0.05 .^{* *}: \mathrm{p}<0.001$.

\begin{tabular}{|c|c|c|c|c|c|c|}
\hline \multicolumn{7}{|c|}{$\underline{\text { Resting } 1 \text { session was compared with other sessions }}$} \\
\hline \multicolumn{7}{|c|}{ Fp1 } \\
\hline & $\mathrm{R} 1 \backslash \mathrm{N}$ & $\mathrm{R} 1 \backslash \mathrm{MV} 1$ & R1\MV2 & R1\MV3 & R1\MV4 & $\mathrm{R} 1 \backslash \mathrm{R} 2$ \\
\hline p-value & $0.000^{* *}$ & $0.000^{* *}$ & $0.000^{* *}$ & $0.000^{* *}$ & $0.000^{* *}$ & 0.524 \\
\hline \multicolumn{7}{|c|}{ Fp2 } \\
\hline & $\mathrm{R} 1 \backslash \mathrm{N}$ & $\mathrm{R} 1 \backslash \mathrm{MV} 1$ & $\mathrm{R} 1 \backslash \mathrm{MV} 2$ & R1\MV3 & R1\MV4 & $\mathrm{R} 1 \backslash \mathrm{R} 2$ \\
\hline p-value & $0.000^{* *}$ & $0.000^{* *}$ & $0.000^{* *}$ & $0.000^{* *}$ & $0.000^{* *}$ & 0.410 \\
\hline \multicolumn{7}{|c|}{$\mathbf{F z}$} \\
\hline & $\mathrm{R} 1 \backslash \mathrm{N}$ & R1\MV1 & R1\MV2 & R1\MV3 & R1\MV4 & $\mathrm{R} 1 \backslash \mathrm{R} 2$ \\
\hline p-value & $0.049^{*}$ & $0.005^{*}$ & $0.000^{* *}$ & $0.009^{*}$ & $0.024^{*}$ & 0.718 \\
\hline \multicolumn{7}{|c|}{ Nature session was compared with other sessions } \\
\hline \multicolumn{7}{|c|}{ Fp1 } \\
\hline & $\mathrm{N} \backslash \mathrm{R} 1$ & N\MV1 & N\MV2 & N\MV3 & N\MV4 & $\mathrm{N} \backslash \mathrm{R} 2$ \\
\hline p-value & $0.000^{* *}$ & $0.000^{* *}$ & $0.000^{* *}$ & $0.000^{* *}$ & $0.005^{*}$ & $0.001^{* *}$ \\
\hline \multicolumn{7}{|c|}{ Fp2 } \\
\hline & $\mathrm{N} \backslash \mathrm{R} 1$ & $\mathrm{~N} \backslash \mathrm{MV} 1$ & N\MV2 & $\mathrm{N} \backslash \mathrm{MV3}$ & N\MV4 & $N \backslash R 2$ \\
\hline p-value & $0.000^{* *}$ & $0.000^{* *}$ & $0.000^{* *}$ & $0.001^{* *}$ & $0.003^{*}$ & $0.000^{* *}$ \\
\hline \multicolumn{7}{|c|}{$\mathbf{F z}$} \\
\hline & $\mathrm{N} \backslash \mathrm{R} 1$ & $\mathrm{~N} \backslash \mathrm{MV} 1$ & N\MV2 & N\MV3 & N\MV4 & $N \backslash R 2$ \\
\hline p-value & $0.049^{*}$ & $0.005^{*}$ & $0.001^{* *}$ & $0.049^{*}$ & 0.124 & $0.072^{*}$ \\
\hline \multicolumn{7}{|c|}{ Comparison between two adjacent sessions } \\
\hline \multicolumn{7}{|c|}{ Fp1 } \\
\hline & $\mathrm{R} 1 \backslash \mathrm{N}$ & $\mathrm{N} \backslash \mathrm{MV} 1$ & MV1\MV2 & MV2\MV3 & MV3\MV4 & MV4\R2 \\
\hline p-value & $0.000^{* *}$ & $0.000^{* *}$ & 0.214 & 0.189 & 0.325 & $0.000^{* *}$ \\
\hline \multicolumn{7}{|c|}{ Fp2 } \\
\hline & $\mathrm{R} 1 \backslash \mathrm{N}$ & $\mathrm{N} \backslash \mathrm{MV} 1$ & MV1\MV2 & MV2\MV3 & MV3\MV4 & MV4\R2 \\
\hline p-value & $0.000^{* *}$ & $0.000^{* *}$ & 0.477 & 0.439 & 0.249 & $0.000^{* *}$ \\
\hline \multicolumn{7}{|c|}{$\mathbf{F z}$} \\
\hline & $\mathrm{R} 1 \backslash \mathrm{N}$ & $\mathrm{N} \backslash \mathrm{MV} 1$ & MV1\MV2 & MV2\MV3 & MV3\MV4 & MV4\R2 \\
\hline p-value & $0.049^{*}$ & $0.005^{* *}$ & 0.198 & 0.099 & 0.622 & $0.049^{*}$ \\
\hline
\end{tabular}

plexity ratios in Resting 1 session were significantly different from that in Nature and any Movie session, and that the complexity ratios in Nature session were significantly different from the Resting and Movie sessions. Besides, the complexity ratios between ant two movies were insignificant. 


\section{Discussion and Conclusions}

We used the SampEn algorithm and introduced a novel complexity ratio to analyze the EEG signals acquired from subjects being resting, watching video clips of Nature scene and Movie trailers. Results showed that the mean complexity ratio in Movie sessions was lower than that in the Resting and Nature sessions. The decreases of mean complexity ratios can be attributed to attention engagement. A previous study also found that the EEG values of SampEn were lower in attention state than in inattention state, and the differences were mainly in Fp1, Fp2, F3, F4 [9]. Therefore we suggest that the complexity ratios decrease when subjects watch Movie sessions reflect that the subjects are in attention to watch movie trailers and the changes of brain status can be detected on their EEG.

It has been reported that fatigue would make the values of SampEn reduce as well [10]. In other words, the EEG signals are less complex during fatigue. To ensure that fatigue was not a major influence in our study, we have observed in Figure 2 that the mean complexity ratio of Movie sessions did not decrease with respect to time. In addition, the mean value of complexity ratios of Resting 2 session was noticeable higher than the previous session (Movie 4), and was almost the same as that of Resting 1 session. This suggests the reductions of complexity ratios in Movie sessions should not be caused by mental fatigue, but by attention.

In short, we compute the mean complexity ratios of the EEG signals acquired from the Resting, Nature, and Movie sessions. The mean complexity ratios of the Movie sessions were significantly lower than that of Nature and Resting sessions, suggesting the mean complexity ratio can be an effective index to differentiate inattention state and attention state when subjects watched movie trailers. Accordingly, we may use the complexity ratio of EEG as an effective index to identify whether a movie trailer can draw people's attention or not.

\section{Acknowledgements}

This study is supported by National Science Council, Chinese Taipei (NSC 101-2221-E-010-004-MY2 \& NSC 101-2314-B-733-001-MY2) and by the project of Center for Dynamical Biomarkers and Translational Medicine, National Central University, Chinese Taipei (NSC 102-2911-I-008-001).

\section{References}

[1] http://www.boxofficemojo.com/yearly/

[2] http://awfj.org/2008/05/07/awfj-opinion-poll-all-about-movie-trailers/

[3] Fisher, C.E., Chin, L. and Klitzman, R. (2010) Defining Neuromarketing: Practices and Professional Challenges. Harvard Review of Psychiatry, 18, 230-237. http://dx.doi.org/10.3109/10673229.2010.496623

[4] Pradeep, A. (2010) The Buying Brain: Secrets for Selling to the Subconscious Mind. John Wiley \& Sons Ltd., Chichester.

[5] Yılmaza, B., Korkmazb, S., Arslanb, D.B., Güngörb, E. and Asyalıc, M.H. Like/Dislike Analysis Using EEG: Determination of Most Discriminative Channels and Frequencies. Computer Methods and Programs in Biomedicine, 113, 705-713. http://dx.doi.org/10.1016/j.cmpb.2013.11.010

[6] Morris, J.D., Klahr, N.J. and Shen, F., et al. (2009) Mapping a Multidimensional Emotion in Response to Television Commercials. Human Brain Mapping, 30, 789-796. http://dx.doi.org/10.1002/hbm.20544

[7] Vecchiato, G., Toppi, J. and Astolfi, L., et al. (2011) Spectral EEG Frontal Asymmetries Correlate with the Experienced Pleasantness of TV Commercial Advertisements. Medical \& Biological Engineering \& Computing, 49, 579583. http://dx.doi.org/10.1007/s11517-011-0747-x

[8] Richman, J.S. and Moorman, J.R. (2000) Physiological Time-Series Analysis Using Approximate Entropy and Sample Entropy. American Journal of Physiology-Heart and Circulatory Physiology, 278, H2039-H2049.

[9] Yan, N., Wang, J., Wei, N. and Zong, L. (2007) Feature Extraction and Classification of Attention Related Electroencephalographic Signals Based on Sample Entropy. Journal-Xian Jiaotong University, 41, 1237.

[10] Tran, Y., Thuraisingham, R.A., Wijesuriya, N., Nguyen, H.T. and Craig, A. (2007) Detecting Neural Change during Stress and Fatigue Effectively: A Comparison of Spectral Analysis and Sample Entropy. Proceedings of the 3rd International IEEE EMBS Conference on Neural Engineering, Hawaii, 2-5 May, 2-5. 